\title{
Modelling and Control by Neural Network of Electric Vehicle Traction System
}

\author{
Hristiyan Kanchev ${ }^{1}$, Nikolay Hinov ${ }^{1}$, Bogdan Gilev ${ }^{2}$, Bruno Francois ${ }^{3}$ \\ ${ }^{I}$ Faculty of Electronic Engineering and Technologies, Technical University of Sofia, \\ ${ }^{2}$ Faculty of Applied Mathematics and Informatics, Technical University of Sofia, \\ 8 "St. Kliment Ohridski" blvd., 1000 Sofia, Bulgaria \\ ${ }^{3}$ Laboratoire d'Electrotechnique et Electronique de Puissance, Ecole Centrale de Lille \\ Cite Scientifique, 59650 Villeneuve d'Ascq, France \\ hkanchev@tu-sofia.bg
}

\begin{abstract}
Modelling and control by neural network of hybrid electric vehicle traction system is presented in this paper. The electric drive is composed by a battery bank and an ultracapacitor connected in parallel through bidirectional DC converters and a Brushless DC Motor driven by a three-phase inverter. In the electric drive control loop is implemented a NARMA neural network. The mechanical model comprises a gearbox and a model of the road-wheel friction force and vehicle aerodynamics. All the masses and inertia are expressed relative to the rotor of the motor. The model is studied by simulations with two driving cycles and an assessment of the available energy from regenerative braking is performed. The percentage of recycled energy from regenerative braking is assessed.
\end{abstract}

Index Terms-Electric vehicle; Neural network; Regenerative braking; Ultracapacitor.

\section{INTRODUCTION}

The main advantage of electric vehicles, compared to internal combustion engine-driven vehicles is the fact that no local pollution is produced by their propulsion system. Although the main drawback of EVs is their autonomy with a fully charged battery they have an average range of up to $200 \mathrm{~km}-250 \mathrm{~km}$ and the recharging process takes several hours. However, the technology is trying to find a solution for improvement of the abovementioned disadvantages. Many authors propose fuel-cell powered hybrid electric vehicles and real-time implementation of optimization techniques for minimization of the vehicle operational cost using dynamic programming, neural networks or fuzzy-logic based control [1]-[6]. In particular, the control strategies proposed in [4] and [5] use neural network for recognition and prediction of the driving cycles to help reducing the equivalent consumption of the vehicle propulsion system. This paper is focused on the modelling and control of an electric vehicle powered by a battery and supercapacitor. A drawback of predictive-control implementations in most of the studies is the uncertainty in user behaviour and in

Manuscript received 10 September, 2017; accepted 2 February, 2018.

This research is funded by the project „Gestion intelligente des flux énergétiques dans des micro- et nano-réseaux" funded by Agence Universitaire de la Francophonie and the Bulgarian National Fund for Scientific Research. particular the prediction of vehicle acceleration and speed during driving. Therefore, in this study the neural network is used for control of the electric motor. In this paper is presented the modelling of a hybrid electric vehicle propulsion system and control by a NARMA neural network of the electric motor. The electric drive is composed by a battery bank and an UltraCapacitor (UC) connected in parallel through bidirectional DC converters. The Brushless DC Motor is driven by a three-phase inverter. The power split between the UC and the electric motor is performed by hierarchical control algorithm that can be implemented into a microcontroller in the vehicle. Such a realization has the advantage of being less complex than predictive control techniques and is less sensitive to the uncertainty in vehicle usage. The mechanical model comprises a gearbox and a model of the road-wheel friction force and vehicle aerodynamics. All the masses and inertia are expressed relative to the rotor of the electric machine. The electric vehicle model is studied by simulations with two driving cycles and an assessment of the available energy from regenerative braking is performed. One of the driving cycles used for simulations is an urban cycle with frequent accelerations and stops and the other is a suburban cycle without any stops.

\section{CONTROL System Structure}

The energy flow diagram of the studied hybrid electric vehicle structure is presented on Fig. 1. It consists of a battery bank (BAT), which is the main power source and an auxiliary source - an UC, serving as energy buffer for acceleration or regenerative braking. Both sources are connected to a DC bus through bidirectional DC-DC converters. This type of hybrid structure is also known as a parallel structure [7]-[9]. By driving, the electric motor can be fed by the battery bank, by the ultracapacitor or by both of them. Inversely, by regenerative braking, power generated by the electrical machine can be fed into the battery bank, into the UC or both. In the studied structure there are seven options for the energy flows:

- The motor is powered by the battery, no power is exchanged with the UC;

- The motor is powered by the battery. The UC is being 
charged by the battery;

- The battery and the UC are delivering power to the motor. The UC is being discharged;

- The UC is supplying power to the motor. No power is exchanged with the battery;

- No power is delivered to the motor (the vehicle is standing still), the UC is being charged by the battery;

- The electric machine is generating power (regenerative braking) and is charging the UC and the battery;

- The electric machine is generating power and is charging the battery, no power is exchanged with the UC.

The control system selects the appropriate energy flows configuration at any moment for proper system operation and optimal use of the energy harvested from regenerative braking. Identification of the system state and allocation of the appropriate power references to the sources are subject to several constraints: maximal charge and discharge current of the battery, of the UC and their state of charge.

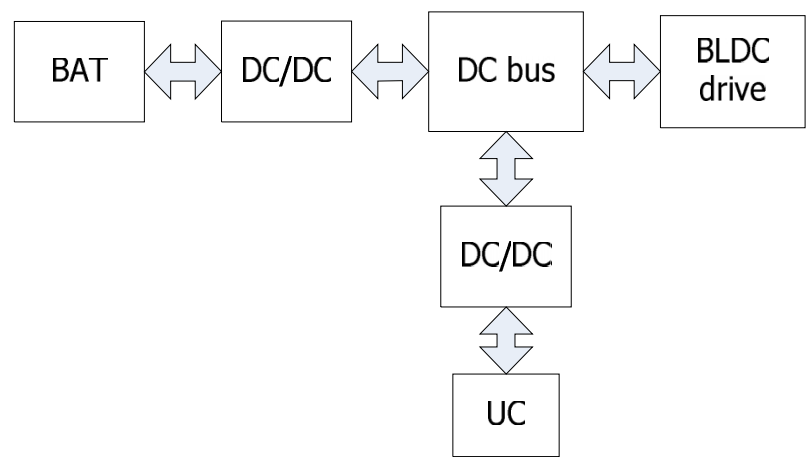

Fig. 1. Energy flows diagram of the studied hybrid electric vehicle structure.

The energy flows management and control of the power converters are performed in two different time scales, therefore the control system is implemented in two levels (Fig. 2): the energy management system that allocates power references to the converters operates at a scale of $0.1 \mathrm{~s}$ and the power converter control systems operate at a timescale of $0.1 \mathrm{~ms}$.

Four distinctive cases of system operation can be considered: by vehicle acceleration, movement with constant speed, regenerative braking and finally when the speed is below a certain value - braking without recuperation. Control of the power flows is performed according to the state of charge of the battery and the UC and the constraints:

- Acceleration: power is delivered from the battery. The $\mathrm{UC}$ is also supplying power if it is charged above a certain voltage level $U_{U C \min }$.

- Movement with constant speed: the vehicle is powered by the battery. There is no power exchanged with the UC.

- Regenerative braking: power generated by the electric machine is returned to the battery and respecting the battery maximum charge current, the excess of power is stored in the UC.

- Mechanical braking: when the vehicle speed falls below a minimal threshold $V_{\text {rmin }}$ regenerative braking stops and mechanical brakes are used.

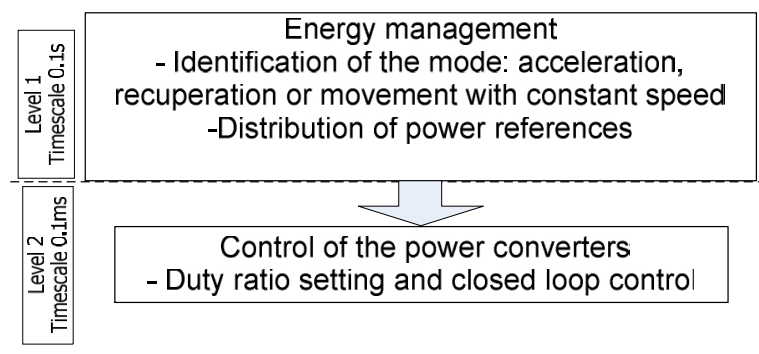

Fig. 2. Hierarchical control structure of the studied system.

\section{ELECTRIC MODEL OF THE SYSTEM}

\section{A. Bidirectional DC Converter Modelling}

A synchronous DC converter (Fig. 3) is obtained from an ordinary Buck converter by replacing the diode with another switch. Both switches operate synchronously - at any moment only one of them is closed and the other one is open. This circuit is interesting also due to its possibility for bidirectional operation (Buck converter in one direction and Boost converter in the other) for energy storage systems and electric vehicles [10], [11]. The combination of a synchronous DC-DC converter and the corresponding control algorithm allows the achievement of highly efficient bidirectional power conversion. In this study losses in converters are neglected: an average model of the converters is implemented. By using this modelling approach, operation of the converters is expressed by their transfer function and is invariant of the circuit parameters.

The equations describing the converter transfer function operating in buck mode are:

$$
\begin{gathered}
\bar{U}_{0}=D \bar{U}_{i}, \\
\bar{I}_{0}=\frac{\bar{I}_{i}}{D},
\end{gathered}
$$

where $D$ is the duty cycle, $\bar{U}_{o}$ and $\bar{I}_{o}$ are the output voltage and current, $\bar{U}_{i}$ and $\bar{I}_{i}$ are the input voltage and current.

By regenerative braking the converter operates as a boost converter and the equations are:

$$
\begin{aligned}
& \bar{U}_{0}=\frac{\bar{U}_{i}}{1-D}, \\
& \bar{I}_{0}=\frac{1-D}{\bar{I}_{i}} .
\end{aligned}
$$

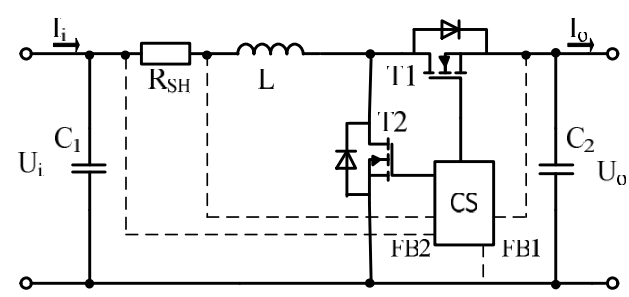

Fig. 3. Synchronous DC-DC converter circuit.

\section{B. Brushless DC (BLDC) Motor}

The voltages and currents are expressed in a rotating reference frame $(d-q)$ [8]. The voltages are expressed by 


$$
\left[\begin{array}{l}
u_{q} \\
u_{d}
\end{array}\right]=\frac{2}{3}\left[\begin{array}{cc}
\cos \left(\theta_{e}\right) & -\sin \left(\theta_{e}\right) \\
\sin \left(\theta_{e}\right) & \cos \left(\theta_{e}\right)
\end{array}\right]\left[\begin{array}{cc}
1 & 1 / 2) \\
0 & -\sqrt{3} / 2)
\end{array}\right]\left[\begin{array}{l}
u_{a b} \\
u_{b c}
\end{array}\right],
$$

where $\theta$ is the rotor position angle, $\theta e=p \theta$ is the field phasor angle and $\mathrm{p}=1$ is the number of pole pairs.

The two-axis currents are then expressed by:

$$
\begin{gathered}
\frac{d i_{q}}{d t}=\frac{1}{L_{q}}\left(u_{q}-R i_{q}-L_{d} \omega_{e} i_{d}-\Phi \omega_{e}\right), \\
\frac{d i_{d}}{d t}=\frac{1}{L_{d}}\left(u_{d}-R i_{d}+L_{q} \omega_{e} i_{q}\right),
\end{gathered}
$$

where $\omega_{e}=p \omega$ is the field angular velocity, $\omega$ is the rotor angular velocity, $\Phi$ is the permanent magnet flux, the active resistance is $R, L_{d}$ and $L_{q}$ are the equivalent inductances of the windings $\left(L_{d}=L_{q}=8.5 \mathrm{mH}\right)$.

Then the three-phase currents in a stationary reference frame $i_{a}, i_{b}$ and $i_{c}$ are calculated by using the inverse Park transformation

$$
\left[\begin{array}{l}
i_{a} \\
i_{b}
\end{array}\right]=\left[\begin{array}{cc}
1 & 0) \\
-1 / 2 & -\sqrt{3} / 2)
\end{array}\right]\left[\begin{array}{cc}
\cos \left(\theta_{e}\right) & \sin \left(\theta_{e}\right) \\
-\sin \left(\theta_{e}\right) & \cos \left(\theta_{e}\right)
\end{array}\right]\left[\begin{array}{c}
i_{q} \\
i_{d}
\end{array}\right] .
$$

And the current $i_{c}$ is

$$
i_{c}=-i_{a}-i_{b}
$$

The machine torque is expressed by the following equation

$$
T_{e}=1,5 p\left(\Phi i_{q}+\left(L_{d}-L_{q}\right) i_{d} i_{q}\right)
$$

\section{Rotor Position Encoder and Decoder}

Switching of the three-phase inverter is implemented by a Hall Effect encoder and decoder. The encoder is composed by three hall sensors (HS_A, B and C) - one for each stator winding. As a result the rotor position and the appropriate polarity of the voltages fed to the stator windings are encoded as a binary number (Table I) which is then sent to the rotor position decoder. The rotor position decoder does exactly the inverse operation: based on the encoder readings it sends signals to the driver circuits of the inverter and in this way ensures adequate switching of the three phase voltages.

TABLE I. HALL EFFECT ENCODER AND DECODER OPERATION.

\begin{tabular}{|c|c|c|c|c|c|}
\hline $\boldsymbol{H} \boldsymbol{S}_{-} \boldsymbol{A}$ & $\boldsymbol{H S} \boldsymbol{B}$ & $\boldsymbol{H S} \boldsymbol{C}$ & $\boldsymbol{V a}$ & $\boldsymbol{V b}$ & $\boldsymbol{V} \boldsymbol{c}$ \\
\hline 0 & 0 & 0 & 0 & 0 & 0 \\
\hline 0 & 0 & 1 & 0 & -1 & +1 \\
\hline 0 & 1 & 0 & -1 & +1 & 0 \\
\hline 0 & 1 & 1 & -1 & 0 & +1 \\
\hline 1 & 0 & 0 & +1 & 0 & -1 \\
\hline 1 & 1 & 0 & 0 & +1 & -1 \\
\hline 1 & 1 & 1 & 0 & 0 & 0 \\
\hline
\end{tabular}

The voltages supplied to the stator windings are:

$$
\mid \begin{aligned}
& u_{a}=\bar{U}_{0} V_{a}, \\
& u_{b}=\bar{U}_{0} V_{b}, \\
& u_{c}=\bar{U}_{0} V_{c},
\end{aligned}
$$

and

$$
\mid \begin{aligned}
& u_{a b}=u_{b}-u_{a} \\
& u_{b c}=u_{c}-u_{b}
\end{aligned}
$$

\section{MechaniCAL MOdEL OF THE SySTEM}

The mechanical stage of the motor and the total inertia of the vehicle traction is expressed relative to the rotor (singlemass mechanical model), according to the following differential equations:

$$
\begin{gathered}
\frac{d \omega}{d t}=\frac{1}{J}\left(-T_{m}+T_{e}-F \omega_{r}\right), \\
\frac{d \theta}{d t}=\omega,
\end{gathered}
$$

where $\omega$ is the rotor angular velocity, $\theta$ is the rotor position angle, $J$ is the total inertia relative to the rotor, $T_{e}$ is the electromagnetic torque of the motor, $F$ is the friction due to the wheel-road contact and $T_{m}$ is the mechanical resistive torque relative to the rotor.

The mechanical model of the closed-loop system in Fig. 4 comprises also the blocks "GearBox", "Wheel" and "Contact Road Wheel". In these blocks the following equations are used:

$$
\begin{gathered}
\omega_{\text {red }}=k \omega, \\
T_{m}=k T_{\text {wheel }}, \\
V_{\text {wheel }}=2 \pi r \omega_{\text {red }}, \\
T_{\text {wheel }}=r F,
\end{gathered}
$$

where $k$ is the gear ratio, $V_{\text {wheel }}$ is the wheel velocity, $T_{\text {wheel }}$ is the wheel torque, $r$ is the wheel radius $(r=0,26 \mathrm{~m})$ and $F$ is the traction force (Fig. 5). Calculation of the traction force is performed in the block "Contact Road Wheel" according to the procedure described in [11].

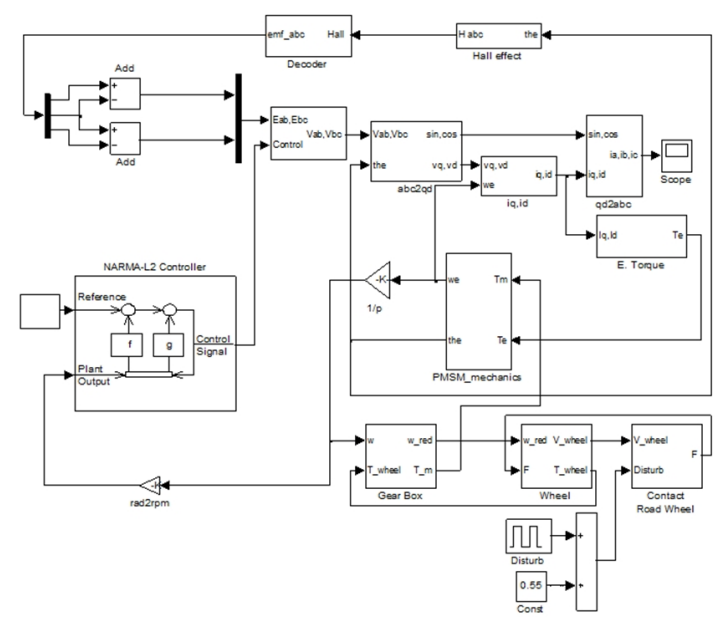

Fig. 4. Model of the closed-loop system in MATLAB. 
The relative disturbance in the wheel velocity is

$$
d(t)=\left\{\begin{array}{l}
0,55 \text { for } 0<t \leq T / 2 \\
0,60 \text { for } T / 2<t \leq T
\end{array}\right.
$$

The mechanical disturbance is cyclic with period $T=$ $0,1 \mathrm{~s}$.

This disturbance simulates flaws in the road surface. Then the actual velocity of the wheel is

$$
V_{1}=V_{\text {whel }} d
$$

The relative velocity wheel-road is:

$$
\begin{gathered}
V_{2}=V_{\text {wheel }}-V_{1}, \\
V_{\text {ratio }}=\frac{V_{2}}{V_{\text {whewl }}+10^{-6}} .
\end{gathered}
$$

The traction force in SI units acting on the wheel is

$$
F=0.9 F_{\text {ratio }}
$$

where the relative traction force is $F_{\text {ratio }}$ (Fig. 5).

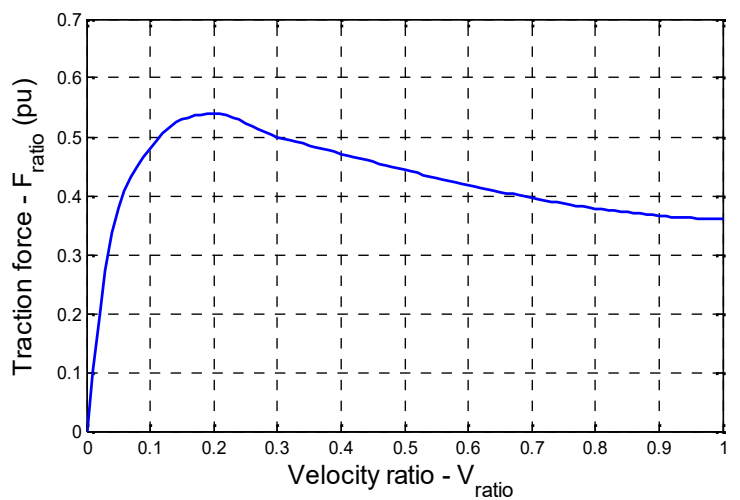

Fig. 5. Model of the road-wheel friction force.

\section{CONTROLLER SYNTHESIS AND NUMERICAL RESULTS}

A NARMA neural network (NN) is implemented in MATLAB [12]-[15]. The implemented NN has two layers: a non-linear and a linear layer. Training of the $\mathrm{NN}$ is performed by batch data. The training data includes simulated trajectories of the system with various time delays.

The plant identification process [14] is performed according to the Plant Identification-NARMA-L2 tool in MATLAB. For plant identification the closed-loop system model (Fig. 4) is transformed into an open-loop system by removing the NARMS-L2-controller. Then the NN is trained by its input - amplification ratio and its target - the motor angular velocity. The corresponding parameters of the $\mathrm{NN}$ are presented on Fig. 6. is

The mathematical function implemented by the $\mathrm{NN}$ model

$$
\begin{gathered}
y(k+1)=f(y(k), y(k-1), \ldots, u(k-1), u(k-2), \ldots)+ \\
\quad+g(y(k), y(k-1), \ldots, u(k-1), u(k-2), \ldots) u(k),
\end{gathered}
$$

where $y$ is the model output, $u$ is the control variable, $f$ and $g$ are approximation functions, synthesized during the $\mathrm{NN}$ training process.

The equation of the control function is then obtained by solution of (23) with variable $u(k)$

$$
\begin{gathered}
u(k)= \\
=\frac{\hat{y}(k+1)-f(y(k), y(k-1), \ldots, u(k-1), u(k-2), \ldots)}{g(y(k), y(k-1), \ldots, u(k-1), u(k-2), \ldots)} .
\end{gathered}
$$

Therefore given the reference signal the system response $u(k)$ is calculated $(24)$ by replacing $y(k+1)$ with the reference signal $\hat{y}(k+1)$.

The NN structure is chosen and trained properly according to the results in Fig. 7 - the plant output corresponds to the NARMA NN output.

With the closed-loop system model (Fig. 4) numerical results are obtained. A similar closed-loop system with PI controller instead and NN controller is realized in [14].

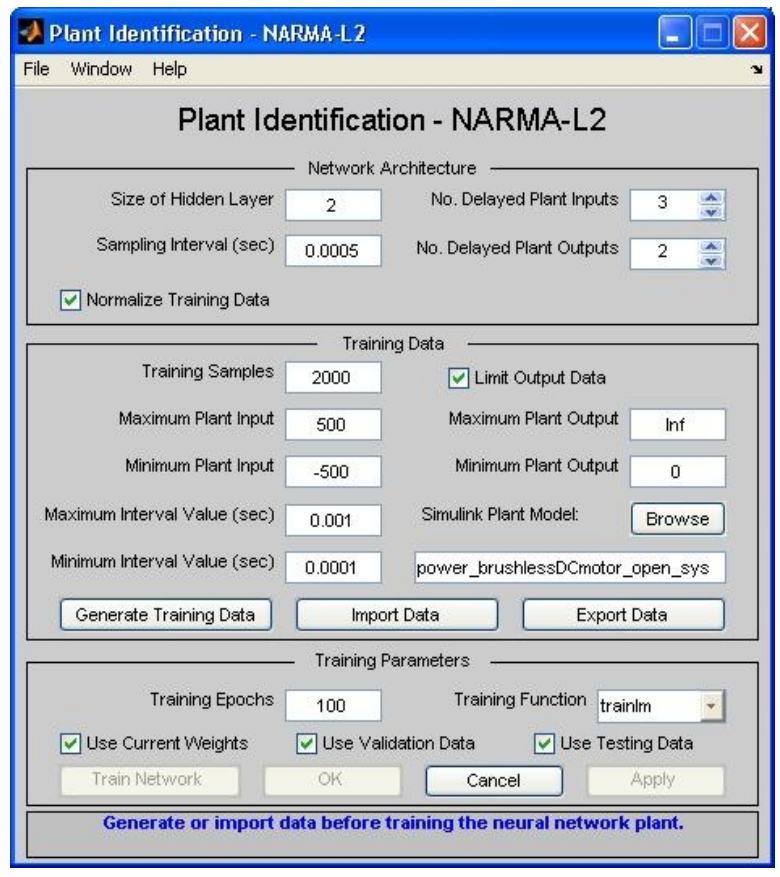

Fig. 6. Parameters of the NARMA-L2 neural network.

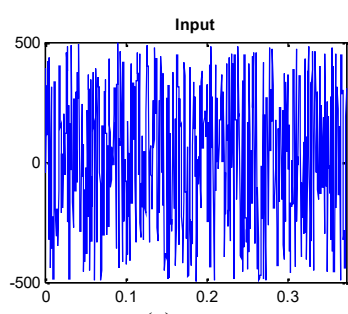

(a)

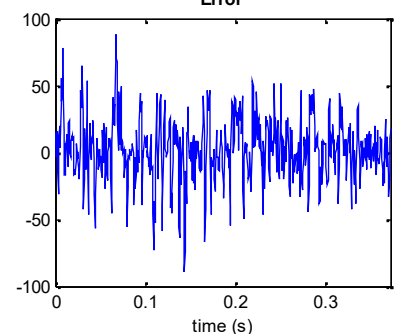

(c)

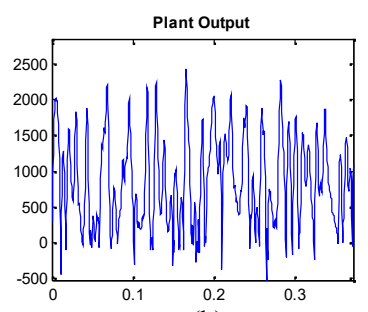

(b)

NN Output

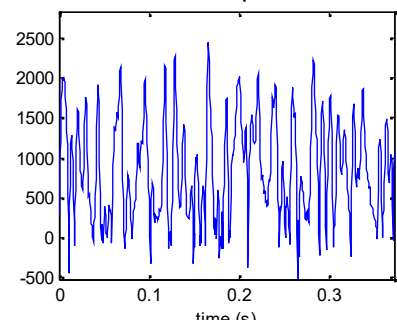

(d)
Fig. 7. Results from the NN training: a) input signal; b) model output; c) error and; d) NN output. 
Simulation results demonstrate that the NN control achieves better robustness and less overshoot than PI control by the same velocity reference. The model is also tested with a torque disturbance added to the motor load, modelled by (17). By equal initial conditions, the NN control achieves shorter duration of the transients and better dynamic response. Another advantage of the NN control is less deviation from the reference signal (Fig. 8). A comparison between PI and NARMA control of this system are presented on Table II. Fine tuning of the PI controller for the actual parameters of the model could lead to better results, but it could hardly outperform the NN-based control. Furthermore, the PI controller is sensitive to changes in the system model (i.e. simulations of a vehicle with different mass or aerodynamics) and requires repetitive fine tuning of the controller. Therefore, another advantage of the $\mathrm{NN}$ control is that training and tuning are performed automatically.

TABLE II. COMPARISON BETWEEN PID AND NARMA CONTROL.

\begin{tabular}{|c|c|c|}
\hline & $\begin{array}{c}\text { Transient response } \\
\text { time }\end{array}$ & Overshoot \\
\hline PI-controller & $0.05 \mathrm{~s}$ & $300 \mathrm{rpm}$ \\
\hline NARMA Controller & $0.01 \mathrm{~s}$ & $30 \mathrm{rpm}$ \\
\hline
\end{tabular}

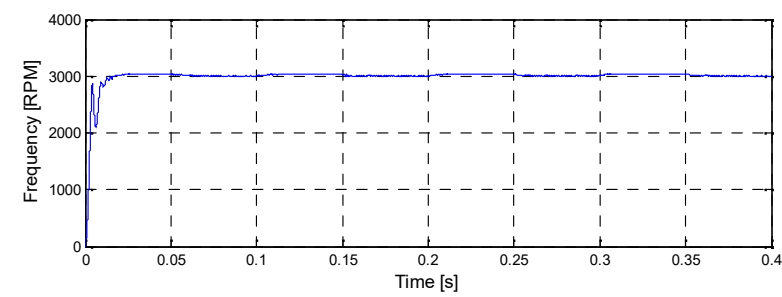

(a)

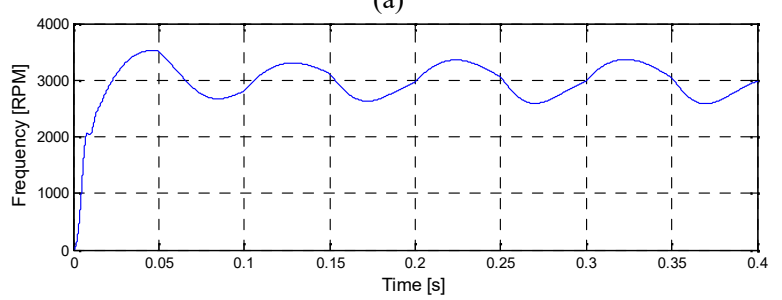

(b)

Fig. 8. Motor angular speed with: a) NN controller and; b)with PI controller.

The presented system model is used for simulations of two typical driving profiles: the first includes a typical city driving profile with numerous accelerations and stops and the second, called "suburban" comprises driving outside the city (Fig. 9).

Both driving cycles are used for simulations of three electric vehicles defined by their mass, frontal area $S$ and drag coefficient $C_{d}$ (Table III).

TABLE III. PARAMETERS OF THE SIMULATED VEHICLES.

\begin{tabular}{|c|c|c|c|c|c|}
\hline $\begin{array}{c}\text { Vehicle } \\
\text { No. }\end{array}$ & $\boldsymbol{m}, \mathbf{k g}$ & $\boldsymbol{S}, \mathbf{m}^{\mathbf{2}}$ & $\boldsymbol{C}_{\boldsymbol{d}}$ & \begin{tabular}{c}
$\boldsymbol{V}_{\max } \boldsymbol{\mathbf { k }} \mathbf{\mathbf { h }}$ \\
\hline 1
\end{tabular} & $\begin{array}{c}\text { Average range, } \\
\mathbf{k m}\end{array}$ \\
\hline 2 & 1200 & 1,5 & 0,35 & 50 & 45 \\
\hline 3 & 1500 & 2,5 & 0,32 & 110 & 70 \\
\hline
\end{tabular}

\section{DISCUSSION}

An estimation of the recycled energy due to regenerative braking is presented in Fig. 10. Simulation results demonstrate that in a city driving cycle the percentage of recycled energy is greater than the suburban cycle due to the often accelerations and stops. Vehicle 1 has a top speed below the minimum allowed speed on motorway, therefore only vehicles 2 and 3 are simulated with HWFET cycle. The percentage of recycled energy of all three vehicles in the CSHVR cycle are similar. A correlation between vehicle mass and the percentage of recycled energy is visible. Although, simulation results demonstrate that recycled energy percentage is mainly dependent on the driving cycle and less dependent on vehicle parameters: in the urban driving cycle the percentage of recycled energy of all three vehicles is in the range of $35 \%-40 \%$. In the suburban cycle also the percentages of both simulated vehicles are similar.

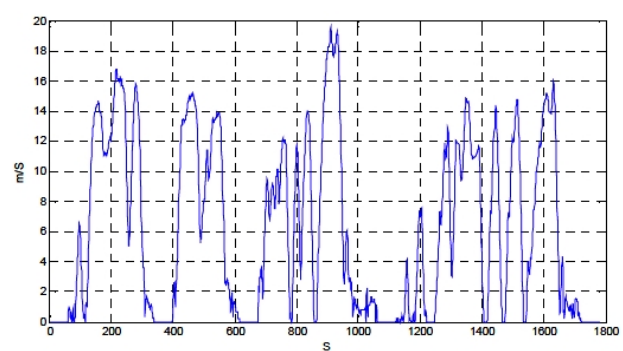

(a)

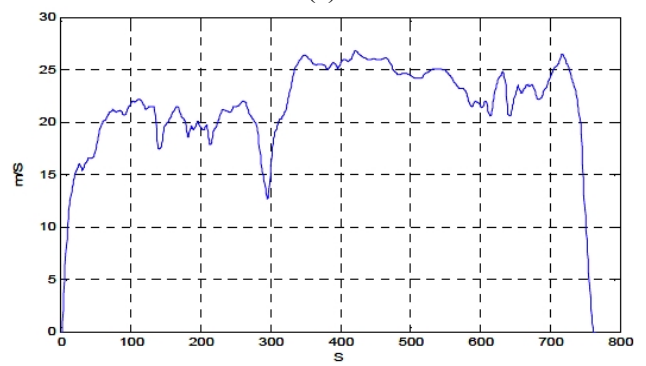

(b)

Fig. 9. Vehicle speed in: a) city driving cycle (CSHVR) and; b) suburban cycle (HWFET)

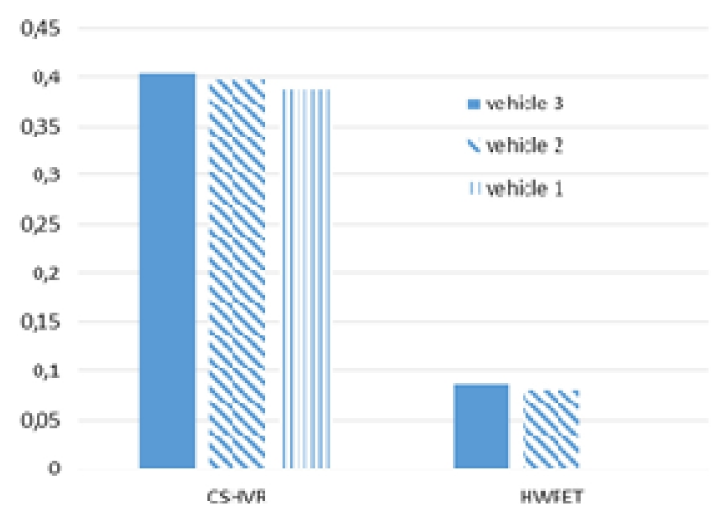

Fig. 10. Recycled energy percentage in the studied driving cycles.

\section{CONCLUSIONS}

Modelling and control by neural network of hybrid electric vehicle traction system is presented in this paper. The electric drive is composed by a $48 \mathrm{~V}$ battery bank, a bidirectional DC converter and Brushless DC Motor driven by a three-phase inverter. In the electric drive control loop is implemented a NARMA neural network. The mechanical model comprises a gearbox, differential and a model of the road-wheel friction force and vehicle aerodynamics. All the masses and inertia are expressed relative units converted to the rotor of the electric machine. Simulation results demonstrate proper operation of the electric drive control 
system.

The electric vehicle model is studied by simulations with two driving cycles and an assessment of the available energy from regenerative braking is performed. The percentage of recycled energy in the city driving cycle is greater than the suburban cycle and is also proportional to the vehicle mass. Furthermore, simulation results demonstrate that recycled energy percentage is mainly dependent on the driving cycle and less dependent on vehicle parameters.

\section{REFERENCES}

[1] S. H. Karaki, R. Jabr, R. Chedid, F. Panik, "Optimal energy management of hybrid fuel cell electric vehicles", SAE Technical Paper, No. 2015-01-1359, 2015, DOI: 10.4271/2015-01-1359

[2] S.H. Karaki, R. Dinnawi, R. Jabr, R. Chedid, F. Panik, "Fuel cell hybrid electric vehicle sizing using ordinal optimization", $S A E$ International Journal of Passenger Cars-Electronic and Electrical Systems, vol. 8, pp. 60-69, 2015. DOI: 10.4271/2015-01-0155.

[3] L. Feldkamp, M. Abou-Nasr, I. V. Kolmanovsky, "Recurrent neural network training for energy management of a mild hybrid electric vehicle with an ultra-capacitor", in IEEE Workshop Computational Intelligence in Vehicles and Vehicular Systems (CIVVS 2009), 2009, pp. 29-36. DOI: 10.1109/CIVVS.2009.4938720.

[4] T. Yi, Z. Xin, Z. Liang, Z. Xinn, "Intelligent energy management based on driving cycle identification using fuzzy neural network", Second Int. Symposium Computational Intelligence and Design (ISCID 2009), 2009, vol. 2, pp. 501-504. DOI: 10.1109/ISCID.2009.271.

[5] C. Majed, S. Karaki, R. Jabr, "Neural network technique for hybrid electric vehicle optimization", in Proc. $18^{\text {th }}$ Mediterranean Electrotechnical Conf. (MELECON 2016), Limmasol, Cyprus, 2016. DOI: 10.1109/MELCON.2016.7495327.
[6] R. Dinnawi, D. Fares, R. Chedid, S. Karaki, R. Jabr, "Optimized energy management system for fuel cell hybrid vehicles", in 17th IEEE Mediterranean Electrotechnical Conf. (MELECON 2014), 2014, pp. 97-102. DOI: 10.1109/MELCON.2014.6820514.

[7] I. Husain, Electric and Hybrid Vehicles Design Fundamentals. New York: CRC press, 2003, ch. 8.

[8] C. C. Chan, "The state of the art of electric, hybrid and fuel cell vehicles", in Proc. of the IEEE, vol. 95, no. 4, 2007, pp. 704-718. DOI: 10.1109/JPROC.2007.892489.

[9] M. Ehsani, Y. Gao, S. E. Gay, A. Emadi, Modern Electric, Hybrid Electric and Fuel Cell Vehicles. New York: CRC Press, ch. 6, 2005.

[10] L. Cheng, P. Acuna, R. P. Aguilera, M. Ciobotaru, J. Jiang, "Model predictive control for DC-DC boost converters with constant switching frequency", IEEE 2nd Annual Southern Power Electronics Conf. (SPEC 2016), pp. 1-6. DOI: 10.1109/SPEC.2016.7846189.

[11] J. N. Verhille1, A. Bouscayrol, P. J. Barre, J. C. Mercieca J. P. Hautier, E. Semail, "Torque tracking strategy for antislip control in railway traction systems with common supplies", 39th IAS Annual Meeting Conf. Record of the 2004 IEEE Industry Applications Conf., 2004, vol. 4, pp. 2738-2745. DOI: 10.1109/IAS.2004.1348862.

[12] Z. B. Jun, J. Lee, T. Lee, C. Won, "Control of IPMSM for Hybrid Electric Commercial Vehicle", in Proc. Vehicle Power and Propulsion Conf., 2010, pp. 1-4. DOI: 10.1109/VPPC.2010.5729118.

[13] D. Grenier, L. A. Dessaint, O. Akhrif, Y. Bonnassieux, B. LePioufle, "Experimental non-linear torque control of a permanent magnet synchronous motor using saliency", IEEE Trans. on Industrial Electronics, vol. 44, no. 5, pp. 680-687, 1997. DOI: 10.1109/41.633471.

[14] I. Inoan, M. Abrudean, "Design of NARMA-L2 neural controller for an induction motor's speed control", Journal of Computer Science and Control Systems, vol. 6, no. 2, pp. 18-21, 2013.

[15] A. Consoli, G. Scarcella, G. Scelba, A. Testa, "Steady-state and transient operation of IPMSMs under maximum torque per ampere control", IEEE Trans. on Industry Applications, vol. 46, pp.121-129, 2010. DOI: 10.1109/TIA. 2009.2036665. 